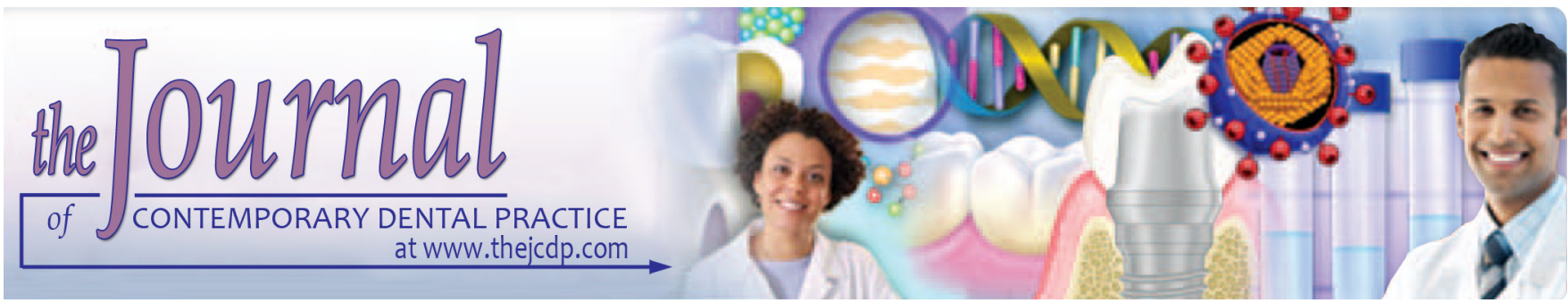

\title{
Effect of Silver Nanoparticles, Zinc Oxide Nanoparticles and Titanium Dioxide Nanoparticles on Microshear Bond Strength to Enamel and Dentin
}

\author{
1'Zahra Jowkar, ${ }^{2}$ Nazbanoo Farpour, ${ }^{3}$ Fatemeh Koohpeima, ${ }^{4}$ Mohammad J Mokhtari, ${ }^{5}$ Fereshteh Shafiei
}

\begin{abstract}
Aim: This study was aimed to evaluate whether antibacterial pretreatment of enamel and dentin with silver nanoparticles (SNPs), zinc oxide nanoparticles (ZNPs) and titanium dioxide nanoparticles (TNPs) has any effect on the microshear bond strength of an etch-and-rinse adhesive system.
\end{abstract}

Materials and methods: Eighty human third molars were randomly assigned to eight subgroups $(n=10)$. Enamel groups included no pretreatment $(E)$, pretreatments with SNPs (ESNP), ZNPs (EZNP) and TNPs (ETNP) before acid etching and adhesive application. Dentinal groups included no pretreatment (D), pretreatments with SNPs (DSNP), ZNPs (DZNP) and TNPs (DTNP). The specimens were bonded by Adper Single Bond and polyvinyl chloride microtubes and were restored with Z250 composite. The bonded surfaces underwent microshear bond strength ( $\mu \mathrm{SBS}$ ) test. Data in megapascal (MPa) were analyzed with the Kruskal-Wallis test and the Mann-Whitney test $(p=0.05)$.

Results: There was not a significant difference among the groups in enamel $(p>0.05)$. There was no significant difference between the application of three nanoparticles and the control group in dentin. However, DSNPs had a higher $\mu$ SBS (25.60 \pm 14.61$)$ than that of the DZNPs and DTNPs groups ( $p=0.03$ and $p=0.001$, respectively). Also, the mean $\mu$ SBS value was lower in dentin groups compared to the respective enamel groups $(p<0.05)$ except for groups DSNPs and ESNPs in which no significant difference was found $(p>0.05)$

\footnotetext{
${ }^{1-3}$ Department of Operative Dentistry, School of Dentistry, Shiraz University of Medical Sciences, Shiraz, Iran

${ }^{4}$ Zarghan Branch, Islamic Azad University, Zarghan, Iran

${ }^{5}$ Department of Operative Dentistry, Oral and Dental Disease Research Centre, School of Dentistry, Shiraz University of Medical Sciences, Shiraz, Iran

Corresponding Author: Zahra Jowkar, Department of Operative Dentistry, School of Dentistry, Shiraz University of Medical Sciences, Shiraz, Iran, Phone: +98-71-36263193, e-mail: zahrajowkar66@gmail.com
}

Conclusion: Pretreatment with SNPs, TNPs, and ZNPs can be suggested to achieve potent antibacterial activities without compromising the bond strength. The best result was obtained for pretreatment with SNPs compared to pretreatment with TNPs or ZNPs in dentin and enamel, albeit the differences were not significant in the enamel groups.

Clinical significance: Effective antibacterial treatment prior to adhesive bonding application is desirable to provide successful restoration if it would not adversely affect the bond strength of the adhesive system. Nanoparticles can be applied to meet this goal.

Keywords: Adhesive bonding, Laboratory research, Microshear bond strength, Nanoparticles.

How to cite this article: Jowkar Z, Farpour N, Koohpeima F, Mokhtari MJ, Shafiei F. Effect of Silver Nanoparticles, Zinc Oxide Nanoparticles and Titanium Dioxide Nanoparticles on Microshear Bond Strength to Enamel and Dentin. J Contemp Dent Pract 2018;19(11):1405-1412.

Source of support: Nil

Conflict of interest: None

\section{INTRODUCTION}

Despite their advantages and their extensive use in the dental clinical practice, resin composites still present some limitations that impair their clinical performance. The principal shortcoming of them include the development of recurrent caries at the composite resintooth interface which is often cited as the main reason for replacement of composite restorations. ${ }^{1,2}$ Nowadays, minimally invasive techniques have been advocated for removing the infected-dentin, leaving behind the caries-affected tissue in the cavity. ${ }^{3}$ Therefore, residual bacteria may still be present in the prepared tooth cavity when the tissue affected by caries is not fully removed and microleakage may allow bacteria to invade the tooth-restoration interfaces during service. ${ }^{4}$ This may lead to the colony growth of bacterial species, especially 
S. mutans, under the restoration, secondary caries and consequently reduced longevity of the restorations. ${ }^{5}$ Therefore, some attempts have been made to hinder bacterial invasion and growth such as incorporating antibacterial agents into adhesives, primers, and composite resins. ${ }^{1,6,7}$ Although different bacteriostatic and bactericide chemicals such as chlorhexidine, Ag-salts and particles, and oxides have been previously incorporated into composite resins to confer antibacterial activity to them, they could potentially jeopardize the composites' physicochemical properties. ${ }^{7,8}$ Moreover, a recent study found that the incorporation of various nanoparticles into adhesive materials may have negative effects on the shear bond strength. ${ }^{9}$ Besides adding active antimicrobial ingredients to the dental materials, another strategy for bacterial reduction is coating surfaces with antibacterial agents to create anti-adhesive surfaces. ${ }^{10,11}$

Recently, nanomaterials referring to the materials with a size of less than $100 \mathrm{~nm}$ have captured more attention from researchers in dentistry because of their unique properties and structures such as small size, large surface area, a large proportion of surface atoms and high surface energy. ${ }^{12}$ In this regard, different metal NPs have been used in various dental branches because of their antibacterial properties. Moreover, bacteria are less likely to develop resistance against metal nanoparticles than a majority of commercially available antibiotics. ${ }^{13}$ Silver nanoparticles (SNPs) has been investigated in dentistry mainly because of their long-term antibacterial property via sustained silver ion release. SNPs have exhibited broad-spectrum antibacterial and antiviral properties in low concentrations related to the multiple antibacterial mechanisms of silver such as the loss of the integrity of bacterial cell membrane and increased cell wall permeability caused by adherence and penetration into the bacterial cell wall, loss of DNA replication ability and inactivation of the vital enzymes of bacteria leading to cell death. ${ }^{14,15}$ Also, it has been shown that SNPs have 25-folds higher antibacterial efficacy than chlorhexidine. ${ }^{16}$ Moreover, biocompatibility of SNPs especially in a lower concentration has been confirmed previously. ${ }^{17}$ A recently published study indicated that an additional pretreatment with SNPs had positive effects on the bond strength of etch-and-rinse and self-etch adhesives with the best results reported for Adper Single Bond and before acid etching. ${ }^{11}$

Similar to SNPs, ZNPs have exhibited antibacterial effects against several types of gram-negative and grampositive bacteria, including S. mutans and Lactobacillus in dental plaque. ${ }^{18,19}$ In fact, ZNPs have demonstrated selective toxicity against bacteria with minimal effects on human cells. ${ }^{20}$ ZNPs provide the antibacterial effect by modification of cell membrane activity and oxidative stress. ${ }^{21}$ Another nanoparticle, which has been recently used in dentistry, is titanium dioxide (TiO2) TNP. Beside their bactericidal effects, TNPs have pleasing color and high biocompatibility. ${ }^{6,22}$ Also, better antibacterial properties compared to chlorhexidine have been shown for TNPs. ${ }^{16}$

Recently, application of metal-based nanoparticles to improve bond strength properties of composite resins has attracted more attention. ${ }^{11,13}$ To the authors' knowledge there are no published studies that address the effect of SNPs, ZNPs and TNPs pretreatments on the bond strength of the composite resin to enamel and dentin. The inherent bactericidal property of NPs has prompted us to investigate the role of SNPs, ZNPs and TNPs pretreatments on the microshear bond strength of the composite resin to enamel and dentin in the present study.

\section{MATERIALS AND METHODS}

After approval of the study design by the ethics committee for research of Shiraz University of Medical Sciences, eighty caries-free extracted human third molars were collected from 20 to 35 -year-old patients, cleaned with a periodontal curette and stored in $0.5 \%$ chloramine solution at $4^{\circ} \mathrm{C}$ for no longer than 1 month until use. The teeth were previously examined under a stereoscopic microscope (Carl Zeiss, Oberkochen, Germany) for the absence of the structural deformities, abrasion, fracture, crack and previous restorations. The roots were removed from the crown in all the specimens. Forty teeth were prepared for testing the $\mu \mathrm{SBS}$ to enamel (E). After preparing $0.5 \mathrm{~mm}$ deep, flat enamel surfaces at the midbuccal aspects of the teeth using diamond fissure burs (Diamond fissure 330; SS White) in a high-speed handpiece under sufficient water cooling, the teeth were embedded in acrylic resin with the buccal surfaces upward and parallel to the base of the resin block. The buccal surfaces were slightly wet-ground with 320grit silicon carbide papers to obtain standardized flat enamel surfaces. A stereoscopic microscope (Carl Zeiss, Oberkochen, Germany) was used to check for the absence of dentin on the enamel surfaces. Another forty teeth were prepared for performing the $\mu \mathrm{SBS}$ tests on dentin (D). Dentin substrate specimens were prepared by sectioning the crowns using a watercooled low-speed cutting machine (Mecatome T201 A, Presi, Grenoble, France) perpendicular to the long axis of the tooth to expose the flat, midcoronal dentin surfaces by removing the occlusal enamel and the superficial dentin. The sectioned teeth were mounted in acrylic resin (Acropars; Marlik Co., Tehran, Iran) with the dentin surfaces oriented perpendicular to the bottom of the mold. To create a uniform smear 


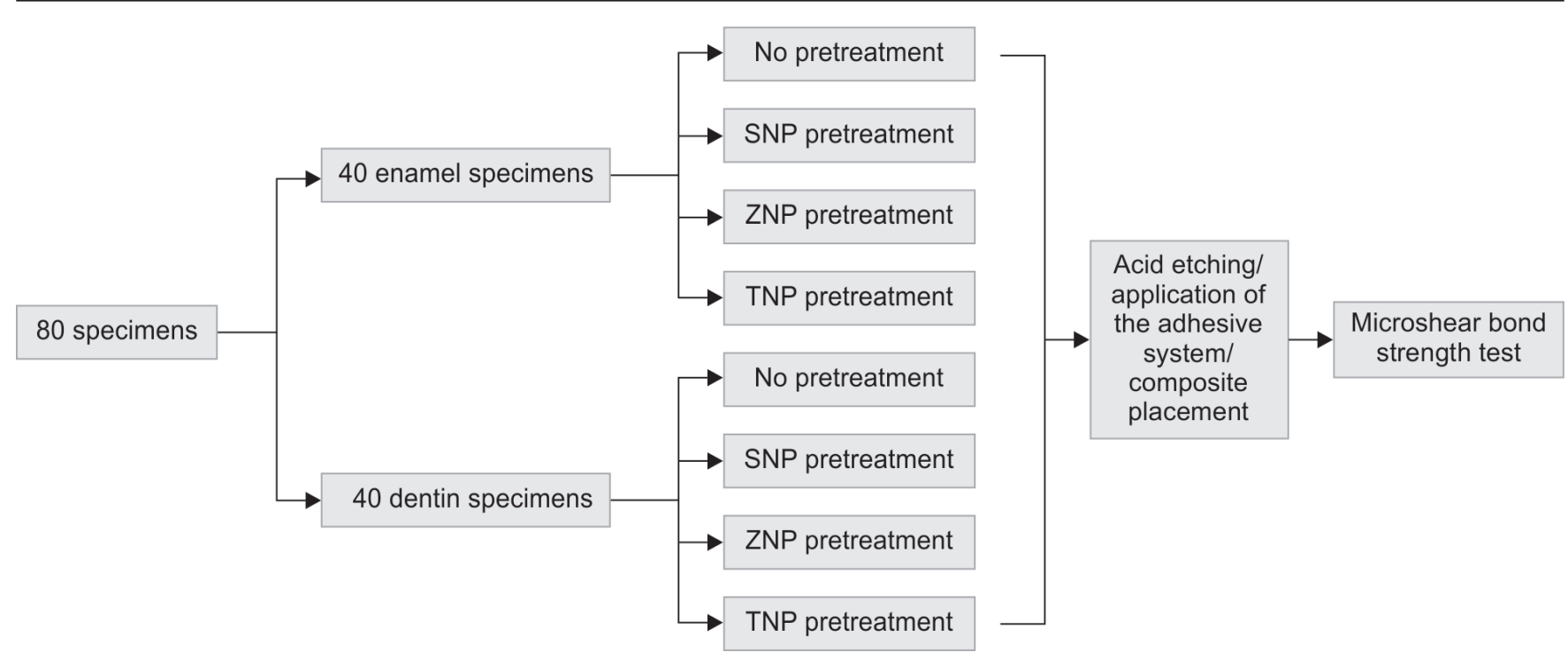

Fig. 1: Diagram of experimental design

layer, the dentin surfaces were slightly wet-ground with 320-grit silicon carbide papers for 1 minute, rinsed and dried with an air-water syringe.

Enamel and dentin samples were randomly divided into four subgroups based on three antibacterial dentin pretreatments with SNPs, ZNPs and TNPs (purchased from US-Nano materials Inc., USA) with an equal number of samples per group $(n=10)$. The sizes of the NPs were 20 $\mathrm{nm}$ for SNPs and TNPs and 10 to $30 \mathrm{~nm}$ for ZNPs. In the control groups (no treatment; $\mathrm{D}$ and $\mathrm{E}$ ), Adper Single Bond (SB, 3M ESPE) adhesive system was used according to the manufacturer's instructions. In groups, ESNPs and DSNPs, surface pretreatment with SNPs was done for one minute before acid etching, and then the dentin or enamel surface was rinsed thoroughly for one minute. Surface pretreatment with ZNPs solution was done in groups EZNPs and DZNPs. Groups ETNPs and DTNPs received surface pretreatment with TNPs solution before acid etching.

Prior to light curing of the adhesives, a piece of translucent polyvinyl chloride microtubes $0.7 \mathrm{~mm}$ in internal diameter and approximately $0.5 \mathrm{~mm}$ height was placed on the bonding surface defined by an adhesive tape with a punched hole over the center of the flattened enamel or dentin surface and subsequently filled with Z250 composite (3M ESPE, St Paul, MN, USA). Light curing was performed using a light curing unit (VIP Junior, Bisco, Schaumburg, IL, USA) at $600 \mathrm{~mW} / \mathrm{cm}^{2}$. The diagram of the experimental design is shown in Figure 1. The bonded specimens were stored in distilled water at $37^{\circ} \mathrm{C}$ for 24 hours and then were placed in a jig attached to a universal testing machine (Instron, Z020. Zwick Roell, Germany). A shear force was applied to each specimen with a direction parallel to the bonded interface at a crosshead speed of $0.5 \mathrm{~mm} /$ minute, as is shown in Figure 2, until failure occurred. The $\mu$ SBS values in MPa were calculated by dividing the recorded load at failure by the bonded surface area. Failure mode analysis was performed by examining the debonded specimens under a stereomicroscope (Carl Zeiss Inc., Oberkochen, Germany) at $\times 40$ and categorized as follows: (A) adhesive failure within the adhesive interfacial zone; (B) cohesive failure in the composite/enamel or dentin; and (C), mixed adhesive failure and cohesive failure. The data were subjected to the Kruskal-Wallis test to compare the groups followed by the Mann-Whitney test for paired comparisons using Statistical Package for the Social Sciences (SPSS) version 17 software (SPSS Inc, Chicago, USA) $(\mathrm{p}<0.05)$.

\section{RESULTS}

Mean $\mu$ SBS and standard deviation (MPa) for the eight groups are presented in Table 1. According to the results

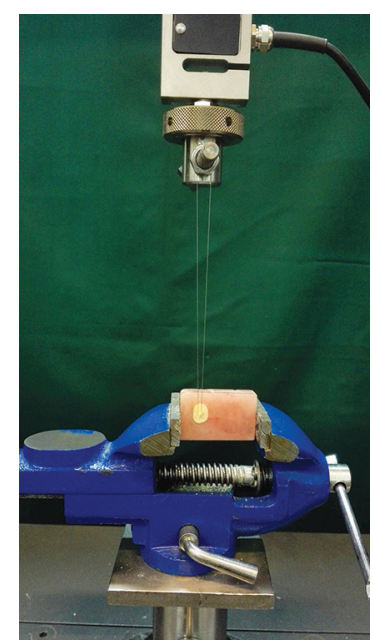

Fig. 2: Stainless steel ligature wire wrapped around the base of resin compositemicro-cylinderbonded to the specimen that is attached to the jig of the universal testing machine 
Table 1: $\mu$ SBS for each group (MPa \pm SD)

\begin{tabular}{lllll}
\hline & \multicolumn{3}{c}{ Groups } \\
\cline { 2 - 5 } & Control (no pretreatment) & SNPs pretreatment & TNPs pretreatment & ZNPs pretreatment \\
\hline Enamel & $46.74 \pm 12.18$ & $40.56 \pm 14.45$ & $34.40 \pm 10.95$ & $39.91 \pm 15.02$ \\
Dentin & $13.70 \pm 5.89$ & $25.60 \pm 14.61$ & $8.80 \pm 3.07$ & $14.76 \pm 10.44$ \\
\hline
\end{tabular}

Table 2: Fracture modes to enamel and dentin

\begin{tabular}{|c|c|c|c|c|c|c|c|c|c|c|c|c|}
\hline & \multicolumn{12}{|c|}{ Groups } \\
\hline & \multicolumn{3}{|c|}{ Control (no pretreatment) } & \multicolumn{3}{|c|}{ SNPs pretreatment } & \multicolumn{3}{|c|}{ TNPs pretreatment } & \multicolumn{3}{|c|}{ ZNPs pretreatment } \\
\hline & $A$ & $\mathrm{C}$ & $\mathrm{M}$ & A & $\mathrm{C}$ & $\mathrm{M}$ & $A$ & $\mathrm{C}$ & M & $A$ & $\mathrm{C}$ & $\mathrm{M}$ \\
\hline Enamel & 1 & 2 & 7 & 2 & 1 & 7 & 1 & 0 & 9 & 2 & 1 & 7 \\
\hline Dentin & 1 & 1 & 8 & 1 & 1 & 8 & 2 & 1 & 7 & 1 & 0 & 9 \\
\hline
\end{tabular}

Modes of failure: A-Adhesive failure; C-Cohesive failure; M-Mixed

of Kruskal-Wallis test, there was not a significant difference among the groups in enamel $(p>0.05)$ meaning that the application of three nanoparticles revealed no adverse effect on $\mu S B S$ to enamel. However, the KruskalWallis test showed significant differences among the four groups in dentin $(\mathrm{p}=0.012)$. Despite higher $\mu \mathrm{SBS}$ obtained in the DSNPs group compared to those of the control group, this difference was not statistically significant $(p>0.05)$. There was no significant difference between the application of three nanoparticles and the control group in dentin. However, the Man-Whitney test revealed that DSNPs had a higher $\mu$ SBS $(25.60 \pm 14.61)$ than that in the DZNPs and DTNPs groups $(\mathrm{p}=0.03$ and $\mathrm{p}=0.001$, respectively). Also, the pairwise comparison showed that $\mu \mathrm{SBS}$ was lower in dentin groups compared to the respective enamel groups $(\mathrm{p}<0.05)$ except for groups DSNPs and ESNPs in which no significant difference was found ( $p>0.05$ ). Moreover, fracture analysis revealed that mixed failure was the most common type of observed failure (Table 2).

\section{DISCUSSION}

Nanotechnology and nanomaterials represent an area of investigation that has recently attracted much attention in dentistry and resulted in opening up new ways to benefit patients. ${ }^{12}$ Metal-based nanoparticles such as SNPs, TNPs, and ZNPs have been used in various medical and dental branches because of their antibacterial properties. ${ }^{6,14,18}$ This study was conducted to evaluate the effect of enamel and dentin pretreatment with SNPs, TNPs, and ZNPs on the $\mu \mathrm{SBS}$ of an etch-and-rinse adhesive. The results of the current study showed that the application of SNPs, ZNPs, and TNPs revealed no adverse effect on $\mu$ SBS to enamel and dentin. Although no significant difference was observed among the application of different nanoparticles in enamel, SNPs showed better results compared to TNPs and ZNPs in dentin. Also, no detrimental visual effect on the color of composite resins was observed for nanoparticles used in this study although the samples were not examined under a stereomicroscope. The mean uSBS for the DSNPs was more than that of the control group, albeit the difference was not significant. It seems that the SNP application had a positive effect on wetting the dentin surface and subsequent infiltration of the bonding agent. Moreover, the least values of mean $\mu$ SBS were observed after pretreatment with TNPs in enamel and dentin although the differences were not significant. The $\mu$ SBS was lower in dentin groups compared to the respective enamel groups $(p<0.05)$ except for the groups DSNPs and ESNPs which did not reveal a significant difference among them.

There are two broad mechanisms for using the antibacterial properties of nanoparticles in the oral cavity to reduce the biofilm formation. The first one is combining dental materials with NPs and the second one is coating surfaces with NPs to prevent microbial adhesion. The second mechanism was used in the current study. ${ }^{11,23,24}$

The microshear bond test has been used in the current study to evaluate the bond strength of the dental adhesive to the tooth structure. The microshear test is a reliable and facile method which has overcome the drawbacks of the macroshear test, including inhomogeneous distribution of stress in the area over which the load is applied, the occurrence of the failure in the dentinal substrate at much lower stresses than the substrate strength and the mixed loading mode. ${ }^{25}$

Residual bacteria resulting from incomplete removal of the caries lesion from cavity walls may lead to pulp damage and recurrent caries. ${ }^{26}$ Less removal of tooth structure and minimal intervention dentistry have become more popular recently leading to the increased possibility of leaving more carious tissues in tooth cavity containing active bacteria. ${ }^{3,4}$ Moreover, studies showing microgaps at tooth-restoration interfaces confirm that a complete sealing of the tooth-restoration interface is difficult to achieve in clinical practice, and microgaps may 
be created at the margins as the results of polymerization shrinkage combined with wear and chewing stresses. ${ }^{27,28}$ Therefore, an antibacterial surface pretreatment directly contacting enamel and dentin surface could be beneficial to help disinfect the prepared tooth cavity, eradicate the residual bacteria and combat the new invading bacteria along the tooth-restoration margins. Considering these facts, different cavity disinfectants such as peroxide or chlorhexidine have been used previously by dental practitioners in the treatment of caries because of the difficulty in determining complete removal of the caries lesion from the prepared cavity. ${ }^{26}$ An important point which should be considered when choosing a cavity disinfectant is that an ideal cavity disinfectant should provide effective antibacterial action without having an adverse effect on the bond strength of adhesive systems to enamel and dentin. ${ }^{25}$ However, it was indicated that pretreatment with traditional cavity disinfectants may negatively affect the bond strength of adhesive systems. ${ }^{29}$ Another concern about the use of cavity traditional disinfectants is that they may not exhibit long-term antibacterial effects or may not completely remove the viable microorganisms in the prepared cavity walls. ${ }^{30}$

Silver is an important broad-spectrum antibacterial, and an antiviral agent which exhibits long-term antibacterial property via sustained silver ion release, a low bacterial resistance compared to antibiotics, good biocompatibility with human cells and a low toxicity. ${ }^{13-15,17}$ The exact bactericidal mechanism of silver is not fully understood. Some possible explanations are as follows: (a) silver causes structural damage in the bacteria by oxygen changing into active oxygen (ROS and hydroxyl radicals), (b) the released biologically active silver ions which can interact with biological molecules inhibit DNA's ability to replicate, (c) The direct contact of the particles with the cell wall results in releasing a very high concentration of silver ions in a small area and killing the cell. The last mechanism is responsible mainly for antibacterial activity of entrapped SNPs in resin materials. ${ }^{14,15,31}$ These properties have encouraged SNP application in dentistry such as incorporation of SNPs into dental resins. ${ }^{5,24}$ An important problem regarding incorporation of SNPs into dental resins is the possible adverse effect of the SNPs on the resin color, mechanical properties, and the polymerization process. ${ }^{31,32}$ Moreover, a much higher antibacterial activity was shown by silver nanoparticles $(25 \mathrm{~nm})$ compared with zinc oxide $(125 \mathrm{~nm})$ and gold $(80 \mathrm{~nm})$ nanoparticles in a previous study which might be attributed to the size of applied nanoparticles. ${ }^{33}$ Although the antimicrobial effect of silver against $\mathrm{S}$. mutans has been previously demonstrated, this effect was not present for the SNPs incorporated into the resin cement. An explanation for this finding is that SNPs have a high propensity for aggregation, which decrease the surface energy and consequently antibacterial effect. Moreover, incorporation of SNPs into dental resins result in their entrapment in the specimens resin and decreased elution of the particles from the specimens after polymerization, making the direct contact of SNPs with the bacteria minimal and consequently no or very small remained antibacterial effect. ${ }^{31}$ Considering this discussion, to prevent microbial adhesion, we applied nanoparticles as surface pretreatment to coat enamel and dentin with nanoparticles and explored the effect of enamel and dentin pretreatment with SNPs, ZNPs, and TNPs on the $\mu S B S$ of an etch-and-rinse adhesive. This pretreatment was aimed to benefit from the antibacterial properties of nanoparticles, and the probable effects of this pretreatment on the bond strength were evaluated in this study. The effect of dentin pretreatment with SNPs on the bond strength of adhesive systems was explored in a previous study. It was reported that SNPs had positive effects on the bond strength of adhesive systems with the best results achieved with Adper Single Bond and before acid etching. Moreover, no adverse effect was seen after application of $1 \mathrm{wt} \%$ SNPs on the color of composite resins. ${ }^{11}$ Based on the findings of the mentioned previous study, the selected concentrations for the nanoparticles in the current study were $1 \%$ to prevent the detrimental effect of these NPs on the color of composite resins. As with the previous study, ${ }_{1}^{15}$ visual observation did not reveal any color change after application of the nanoparticles with $1 \mathrm{wt} \%$ concentrations in this study.

Another nanoparticle which was used in this study was ZNP. Zinc oxide has a proper antibacterial activity which is improved by converting into nanoparticles because of the increased surface-to-volume ratio of the nanoparticles. ${ }^{34}$ The antibacterial mechanism of ZNPs is related to modified cell membrane activity and oxidative stress resulting from the generation of active oxygen species such as $\mathrm{H}_{2} \mathrm{O}_{2}$ that inhibit bacterial growth. ${ }^{21}$ The leaching of $\mathrm{Zn}_{2}+$ into the growth media is another antibacterial mechanism that interferes with the bacterial metabolism by displacing $\mathrm{Mg} 2+{ }^{35} \mathrm{Zinc}$ oxide can also hinder the dental plaque acid production by inhibiting Lactobacillus and Streptococcus mutans although a higher concentration compared to NAPs was required for efficacy. ${ }^{18,19,33}$ Moreover, it has been reported that zinc has an inhibitory effect on the activity of matrix metalloproteinases (MMPs) which play roles in degradation of dentin collagen. ${ }^{36}$ Osorio et al. showed a much longer effect on reducing collagen degradation in demineralized human dentin for zinc oxide (three weeks) as compared to that of chlorhexidine, 
which was short-term. They also found that zinc had no adverse effect on bond strength to dentin which was per the result of the present study. ${ }^{37}$ Zinc oxide also can stimulate a metabolic effect in hard tissue mineralization and inhibit dentin demineralization. ${ }^{38,39}$ Besides, a durable and strong bond at the resin/dentin interface was reported for zinc by decreasing collagen degeneration. ${ }^{36}$ It was also shown that $1.23 \%$ and $13 \%$ concentrations of zinc oxide nanoparticles for bonding orthodontic brackets were able to decrease decalcification resulting from orthodontic treatment. ${ }^{40}$ Additionally, composite resins containing silver nanoparticles or zinc oxide nanoparticles demonstrated higher antibacterial activity against Streptococcus mutans and Lactobacillus compared to the control group in a previous study. ${ }^{34}$ In the present study, no adverse effects on $\mu$ SBS to dentin and enamel and composite resin color were observed after surface pretreatment with ZNPs. Therefore, enamel and dentin surface pretreatment with ZNPs can be suggested to benefit from the positive antibacterial effects of ZNPs.

Another nanoparticle which was used in this study was the TNP. The bactericidal mechanism of $\mathrm{TiO} 2$ is the production of free radicals $\left(\mathrm{HO} \bullet\right.$ and $\left.\mathrm{O}_{2} \bullet-\right)$ which are strong oxidants with the capability to induce oxidative damage in the cell walls of microorganisms. ${ }^{41}$ Good antiadhesive properties against Streptococcus mutans were also reported for TNPs in a previous study. ${ }^{42}$ It was shown that incorporating $\mathrm{TiO}_{2}$ nanoparticles into composite resins conferred antibacterial properties to them. However, the mean shear bond strength of composite containing $10 \%$ NPs was lower than that of the control group. ${ }^{43}$ Titanium dioxide $\left(\mathrm{TiO}_{2}\right)$ nanoparticles exhibit better antibacterial properties compared to chlorhexidine. Moreover, TNPs are suggested for preventing white spot formation because bacteria are less likely to develop resistance against TNPs. ${ }^{13}$ In the present study, enamel and dentin surfaces pretreated with TNPs presented the lowest values of $\mu$ SBS, albeit the differences were not significant to the control groups. This finding can be explained by the fact that because of the high surface energy and the resultant strong aggregation of the TNPs, dispersion of TNPs is difficult and this phenomenon directly affects their antimicrobial and physiochemical properties. ${ }^{42}$

Based on the results of the present study, the dentin pretreatment with SNPs showed a statistically significant improvement in adhesive strength compared with the groups that use other nanoparticles as dentin pretreatment. The same result was observed for enamel, albeit the differences among different nanoparticles were not significant. This result can be attributed to the water-based character of SNPs that may provide an increase in the surface tension of the dentin substrate and help inadequate penetration of the adhesive system through the etched dentin. ${ }^{44}$ Another explanation for this finding may be due to the capability of silver to form silver compounds with chloride, phosphate, oxide, and proteins that have relatively low solubility within dentinal tubules which may lead to a durable gradual release of slight silver ions. This phenomenon may provide long-term antibacterial efficacy in the adhesivetooth interface. ${ }^{45}$ Moreover, higher dentin bond strength for SNPs compared to the ZNPs and TNPs might be attributed to the different chemical and colloidal stability of the NPs, charge of the NPs, morphologies, aggregation stability and surface-to-volume ratio of the NPs which leads to different interactions with enamel and dentin. Additionally, the differences in bond strength among the three groups pretreated with the three nanoparticles and the control group were not significant.

Thus, the behavior of different nanoparticles, their interaction with enamel and dentin and the adhesion protocols proposed by this study have to be further investigated, especially for their antibacterial and mechanical properties and toxicity, to be securely used in clinical practice. This study has some limitations. First, it was an in vitro study, and the results of the present study should be confirmed in future in vivo studies. Besides, only one adhesive system and three nanoparticles were used in this study, and we did not investigate the long-term bond strength properties, antibacterial and anti-caries effects of the nanoparticles. Therefore, further in vitro and in vivo studies are needed to investigate the effects of SNPs, TNPs and ZNPs pretreatment on enamel and dentin bond durability and the long-term antibacterial and anti-caries efficacy of these nanoparticles using various adhesive systems, composites, and glass ionomer cement. Moreover, the probable release of nanoparticles into oral cavity and saliva were not evaluated in this study, and they should be investigated in future.

\section{CONCLUSION}

Based on the result of this study, pretreatment with SNPs, TNPs, and ZNPs can be suggested to achieve potent antibacterial activities without compromising the bond strength. The best result was obtained for pretreatment with SNPs compared to pretreatment with TNPs or ZNPs in dentin and enamel, albeit the differences were not significant in the enamel groups.

\section{CLINICAL SIGNIFICANCE}

Effective antibacterial treatment prior to adhesive bonding application is desirable to provide successful restoration if it would not adversely affect the bond strength of the adhesive system. Nanoparticles can be applied to meet this goal. 


\section{ACKNOWLEDGMENTS}

Authors would like to thank the Vice-Chancellery of Research Shiraz University of Medical Science for supporting this research (Number: 1396-01-03-14624). This article is based on the thesis by Dr Farpour. The authors also thank Dr Vosough and Mohammad Salehi of the Dental Research Development Center, of the School of Dentistry for the statistical analysis.

\section{REFERENCES}

1. Melo MA, Cheng L, Weir MD, Hsia RC, Rodrigues LK, Xu $\mathrm{HH}$. Novel dental adhesive containing antibacterial agents and calcium phosphate nanoparticles. J Biomed Mater Res Part B, Applied biomaterials. 2013;101(4):620-629.

2. Sakaguchi RL. Review of the current status and challenges for dental posterior restorative composites: clinical, chemistry, and physical behavior considerations. Summary of discussion from the Portland Composites Symposium (POCOS) June 17-19, 2004, Oregon Health and Science University, Portland, Oregon. Dent Mater. 2005;21(1):3-6.

3. de Almeida Neves A, Coutinho E, Cardoso MV, Lambrechts P, Van Meerbeek B. Current concepts and techniques for caries excavation and adhesion to residual dentin. J Adhes Dent. 2011;13(1):7-22.

4. Li F, Chen J, Chai Z, Zhang L, Xiao Y, Fang M, et al. Effects of a dental adhesive incorporating antibacterial monomer on the growth, adherence and membrane integrity of Streptococcus mutans. J Dent. 2009;37(4):289-296.

5. Kasraei S, Sami L, Hendi S, AliKhani M-Y, Rezaei-Soufi L, Khamverdi Z. Antibacterial properties of composite resins incorporating silver and zinc oxide nanoparticles on Streptococcus mutans and Lactobacillus. Restor Dent Endod. 2014;39(2):109-114

6. Xia Y, Zhang F, Xie H, Gu N. Nanoparticle-reinforced resinbased dental composites. J Dent. 2008;36(6):450-455.

7. Cheng L, Weir MD, Xu HH, Antonucci JM, Lin NJ, LinGibson S, et al. Effect of amorphous calcium phosphate and silver nanocomposites on dental plaque microcosm biofilms. J Biomed Mater Res Part B, Applied biomaterials. 2012;100(5):1378-1386.

8. Miki S, Kitagawa H, Kitagawa R, Kiba W, Hayashi M, Imazato S. Antibacterial activity of resin composites containing surface pre-reacted glass-ionomer (S-PRG) filler. Dent Mater. 2016;32(9):1095-1102.

9. Reddy AK, Kambalyal PB, Patil SR, Vankhre M, Khan MYA, Kumar TR. Comparative evaluation and influence on shear bond strength of incorporating silver, zinc oxide, and titanium dioxide nanoparticles in orthodontic adhesive. J Orthodont Sci. 2016;5(4):127.

10. Mohamed Hamouda I. Current perspectives of nanoparticles in medical and dental biomaterials. J Biomed Res. 2012;26(3):143-151.

11. Fatemeh K, Mohammad Javad M, Samaneh K. The effect of silver nanoparticles on composite shear bond strength to dentin with different adhesion protocols. J Appl Oral Sci. 2017;25:367-373.

12. Li X, Cui R, Liu W, Sun L, Yu B, Fan Y, et al. The Use of Nanoscaled Fibers or Tubes to Improve Biocompatibility and Bioactivity of Biomedical Materials. J Nanomater. 2013;2013:16.
13. Borzabadi-Farahani A, Borzabadi E, Lynch E. Nanoparticles in orthodontics, a review of antimicrobial and anti-caries applications. Acta Odontol Scand. 2014;72(6):413-417.

14. Rai MK, Deshmukh SD, Ingle AP, Gade AK. Silver nanoparticles: the powerful nanoweapon against multidrug-resistant bacteria. J Appl Microbiol. 2012;112(5):841-852.

15. Rai M, Yadav A, Gade A. Silver nanoparticles as a new generation of antimicrobials. Biotechnol Adv. 2009;27(1):76-83.

16. Besinis A, De Peralta T, Handy RD. The antibacterial effects of silver, titanium dioxide and silica dioxide nanoparticles compared to the dental disinfectant chlorhexidine on Streptococcus mutans using a suite of bioassays. Nanotoxicology. 2014;8(1):1-16.

17. Gomes-Filho JE, Silva FO, Watanabe S, Cintra LT, Tendoro KV, Dalto LG, et al. Tissue reaction to silver nanoparticles dispersion as an alternative irrigating solution. J Endod. 2010;36(10):1698-1702.

18. Jones N, Ray B, Ranjit KT, Manna AC. Antibacterial activity of $\mathrm{ZnO}$ nanoparticle suspensions on a broad spectrum of microorganisms. FEMS Microbiol Lett. 2008;279(1):71-76.

19. Hirota K, Sugimoto M, Kato M, Tsukagoshi K, Tanigawa T, Sugimoto H. Preparation of zinc oxide ceramics with a sustainable antibacterial activity under dark conditions. Ceram Int. 2010;36(2):497-506.

20. Sharma V, Shukla RK, Saxena N, Parmar D, Das M, Dhawan A. DNA damaging potential of zinc oxide nanoparticles in human epidermal cells. Toxicol Lett. 2009;185(3):211-218.

21. Xie $Y$, He Y, Irwin PL, Jin T, Shi X. Antibacterial activity and mechanism of action of zinc oxide nanoparticles against Campylobacter jejuni. Appl Environ Microbiol. 2011;77(7):23252331.

22. Heravi F, Ramezani M, Poosti M, Hosseini M, Shajiei A, Ahrari F. In Vitro Cytotoxicity Assessment of an Orthodontic Composite Containing Titanium-dioxide Nano-particles. J Dent Res, Dental Clinics, Dental Prospects. 2013;7(4):192-198.

23. Mohamed Hamouda I. Current perspectives of nanoparticles in medical and dental biomaterials. J Biomed Res. 2012;26(3):143-151.

24. Reddy AK, Kambalyal PB, Patil SR, Vankhre M, Khan MY, Kumar TR. Comparative evaluation and influence on shear bond strength of incorporating silver, zinc oxide, and titanium dioxide nanoparticles in orthodontic adhesive. J Orthod Sci. 2016;5(4):127-131.

25. Sadat-Shojai M, Atai M, Nodehi A, Khanlar LN. Hydroxyapatite nanorods as novel fillers for improving the properties of dental adhesives: Synthesis and application. Dent Mater. 2010;26(5):471-482.

26. Imazato S, Kuramoto A, Takahashi Y, Ebisu S, Peters MC. In vitro antibacterial effects of the dentin primer of Clearfil Protect Bond. Dent Mater. 2006;22(6):527-532.

27. Duarte SJ, Lolato AL, de Freitas CRB, Dinelli W. SEM analysis of internal adaptation of adhesive restorations after contamination with saliva. J Adhes Dent. 2005;7(1):51-56.

28. Loguercio AD, Reis A, Bortoli G, Patzlaft R, Kenshima S, Filho LER, et al. Influence of Adhesive Systems on Interfacial Dentin Gap Formation In Vitro. Oper Dent. 2006;31(4):431-41.

29. Ercan E, Erdemir A, Zorba YO, Eldeniz AU, Dalli M, Ince $B$, et al. Effect of different cavity disinfectants on shear bond strength of composite resin to dentin. J Adhes Dent. 2009;11(5):343-346.

30. Borges FMC, de Melo MAS, Lima JPM, Zanin ICJ, Rodrigues LKA. Antimicrobial effect of chlorhexidine digluconate 
in dentin: In vitro and in situ study. J Conserv Dent : JCD. 2012;15(1):22-26.

31. Mona Riad AYH, Omnia A. Elhiny, Ghada A. Salem. Evaluation of the Shear Bond Strength of Orthodontic Adhesive System Containing Antimicrobial Silver Nano Particles on Bonding of Metal Brackets to Enamel. Life Sci J. 2015;12(12):27-34.

32. Cheng L, Weir MD, Xu HHK, Antonucci JM, Kraigsley AM, Lin NJ, et al. Antibacterial amorphous calcium phosphate nanocomposites with a quaternary ammonium dimethacrylate and silver nanoparticles. Dent Mater. 2012;28(5):561-572.

33. Hernández-Sierra JF, Ruiz F, Cruz Pena DC, Martínez-Gutiérrez F, Martínez AE, de Jesús Pozos Guillén A, et al. The antimicrobial sensitivity of Streptococcus mutans to nanoparticles of silver, zinc oxide, and gold. Nanomedicine. 2008;4(3):237-240.

34. Li F, Weir MD, Chen J, Xu HH. Comparison of quaternary ammonium-containing with nano-silver-containing adhesive in antibacterial properties and cytotoxicity. Dent Mater. 2013;29(4):450-461.

35. Gu H, Fan D, Gao J, Zou W, Peng Z, Zhao Z, et al. Effect of $\mathrm{ZnCl} 2$ on plaque growth and biofilm vitality. Arch Oral Biol. 2012;57(4):369-375.

36. Raquel O, Monica Y, Estrella O, S. RJ, Manuel T. Zinc-doped dentin adhesive for collagen protection at the hybrid layer. Eur J Oral Sci. 2011;119(5):401-410.

37. Osorio R, Yamauti M, Osorio E, Ruiz-Requena ME, Pashley DH, Tay FR, et al. Zinc reduces collagen degradation in demineralized human dentin explants. J Dent. 2011;39(2):148-153.

38. Hoppe A, Guldal NS, Boccaccini AR. A review of the biological response to ionic dissolution products from bio- active glasses and glass-ceramics. Biomaterials. 2011;32(11): 2757-2774.

39. Takatsuka T, Tanaka K, Iijima Y. Inhibition of dentine demineralization by zinc oxide: In vitro and in situ studies. Dent Mater. 2005;21(12):1170-1177.

40. Spencer CG, Campbell PM, Buschang PH, Cai J, Honeyman AL. Antimicrobial Effects of Zinc Oxide in an Orthodontic Bonding Agent. Angle Orthod. 2009;79(2):317-322.

41. Shirai R, Miura T, Yoshida A, Yoshino F, Ito T, Yoshinari M, et al. Antimicrobial effect of titanium dioxide after ultraviolet irradiation against periodontal pathogen. Dent Mater J. 2016;35(3):511-516.

42. Cao B, Wang Y, Li N, Liu B, Zhang Y. Preparation of an orthodontic bracket coated with an nitrogen-doped $\mathrm{TiO} 2-\mathrm{xNy}$ thin film and examination of its antimicrobial performance. Dent Mater J. 2013; 32(2):311-316.

43. Sodagar A, Akhoundi MSA, Bahador A, Jalali YF, Behzadi $\mathrm{Z}$, Elhaminejad $\mathrm{F}$, et al. Effect of $\mathrm{TiO} 2$ nanoparticles incorporation on antibacterial properties and shear bond strength of dental composite used in Orthodontics. Dental Press J Orthod. 2017;22(5):67-74.

44. Blöcher S, Frankenberger R, Hellak A, Schauseil M, Roggendorf MJ, Korbmacher-Steiner HM. Effect on enamel shear bond strength of adding microsilver and nanosilver particles to the primer of an orthodontic adhesive. BMC Oral Health. 2015;15:42.

45. Mei ML, Ito L, Cao Y, Li QL, Chu CH, Lo EC. The inhibitory effects of silver diamine fluorides on cysteine cathepsins. J Dent. 2014;42(3):329-335. 